\section{Screening Li-Ion Batteries for Internal Shorts}

\author{
Eric Darcy \\ NASA-Johnson Space Center \\ Houston, TX USA
}

\section{Introduction}

The extremely high cost of aerospace battery failures due to internal shorts makes it essential that their occurrence be very rare, if not eliminated altogether. With Li-ion cells/batteries, the potentially catastrophic safety hazard that some internal shorts present adds additional incentive for prevention. Prevention can be achieved by design, manufacturing measures, and testing.

Specifically for NASA's spacesuit application, a Li-ion polymer pouch cell battery design is in its final stages of production. One of the 20 flight batteries fabricated and tested developed a cell internal short which did not present a safety hazard, but has required revisiting the entire manufacturing and testing process. Herein are the details of the failure investigation that followed to get to root cause of the internal short and the corrective actions that will be taken. The resulting lessons learned are applicable to most Li-ion battery applications.

\section{Spacesuit Battery Design}

This $20 \mathrm{~V}, 37 \mathrm{Ah}$ spacesuit battery (Fig. 1) consists of 5 cell modules connected in series through a printed circuit board and housed in an aluminum box. This battery has two capped external connectors, J1, which connects to spacesuit electrical bus, and $\mathrm{J} 2$, which connects to an external charger, that can rebalance the state of charge (SoC) of each cell module (Fig. 2). Each cell module consists of 5 pouch cells $(7.4 \mathrm{Ah}$ ) whose tabs are welded to a pair of terminal blocks. This places the 5 cells in parallel electrically (Fig. 3). Each cell is roughly $100 \times 75 \times 9 \mathrm{~mm}$, and its electrochemistry consists of graphite, cobaltate, and $\mathrm{LiPF}_{6}$ salt (Fig. 4). The cell design consists of a single anode and cathode, and two layers of separator laminated together as a $\sim 1 \mathrm{~m} \mathrm{x} \sim 100$ $\mathrm{mm}$ cell. The substrate foil for the anode and cathode is laminated with active material only on one side. The laminated cell assembly is then z-folded 15 times into a $\sim 9 \mathrm{~mm}$ thick cell stack. The cell stack is then sealed in a plastic-aluminum laminate pouch material. The five cells are stacked and sealed in a similar plastic-aluminum laminate outer pouch. The fragile assembly is then placed in a mold to be potted with urethane foam to become a solid 37Ah cell module brick. The entire battery assembly, including the cells, is designed and manufactured at Electrovaya Corp., in Mississauga, Canada. The cell and cell module acceptance is also performed by them, while the majority of the battery acceptance testing is done at NASA-JSC.

\section{Current Internal Short Controls}

Each cell is manufactured and extensively screened for visual, physical, and other defects such as loss of hermeticity, soft shorts, high self-discharge rate, anomalous capacity and impedance performance. The cell module acceptance testing consists of two capacity and impedance performance cycles. The battery acceptance testing consists of visual, physical, capacity, and impedance verifications before and after thermal cycling and random vibration testing.

For battery $\mathrm{s} / \mathrm{n} 1010$, all the verifications were completed in Oct 2005 and indicative of a healthy battery.
After recharging it to $30 \% \mathrm{SoC}$, the unit was placed in its shipping container until it was called to support a charger test in Feb 2006. At this time, one of its cell module was found to have an OCV of $\sim 10 \mathrm{mV}$, while the other 4 modules where at their nominal $3.78 \mathrm{~V}(30 \% \mathrm{SoC})$.

\section{Plausible Root Causes}

Careful, deliberate, non-destructive, and destructive physical examinations (DPA) isolated the short to one of the cell pouches within the defective cell module, specifically, the outer most cell \#5 in the stack (Figs. 5 and 6). Specifically, both cell bottom corners show evidence of high heat affected zones with their centers located very near the corners (Fig. 7). There appears to be two "ground zeros" for the short, which when fed by the energy of all five cells caused intense heating, high enough to melt the copper foil $\left(1084^{\circ} \mathrm{C}\right)$ in microscopic locations (Fig. 8). This heat damaged the bottom corners of the adjacent cell \#4. No evidence of separator failure or foreign object debris (FOD) was found. This leaves electrode misalignment, native contamination, cold flow through the plastic-aluminum laminate, and pouch seal fold impingement as remaining possible root causes aggravated by the thermal cycling or/and vibration.

During the DPA, non-uniformities in the thickness of the urethane foam potting around the cell module were found in the layers adjacent to the affected bottom corners of cell \#4 and \#5 (Fig. 9). Thin and thick spots could have left the stack of pouch cells unsupported at the corners allowing relative motion during vibration.

This could have aggravated to any of the four root causes mentioned earlier.

Vibration could have stressed the unsupported electrode substrate folds and cause them to tear (Fig. 10). This in turn could have damaged the separator and allowed misalignment of the electrode folds. Similarly, vibration could have caused some active electrode material to break loose and contaminate the substrate fold area, where no active material should be present. This contamination could have cause separator damage and a short. Also, impingement of the cell stack corner against the laminate pouch could have causes its plastic outer layers to cold flow until the folded cell stack made direct contact with the aluminum layer in the pouch.

Alternatively, the folded pouch seal on the external edges and corners of the cells could have damaged the cell pouch laminate material at its corner where the pouch is drawn (stretched) to form a cup shape for the cell stack. This is particularly likely for cell $\# 5$, whose edge seal is doubly folded in the direction of cell \#4, and its resultant corner seal fold can result in a very stiff and sharp point (Fig. 11).

The key facts with which the mechanism of this internal short must be consistent are as follows;

a) The 1 minute/axis random vibration test was performed with a $\sim 2 \mathrm{~A}$ current load and with an oscilloscope set to trigger and record any voltage step change $>12 \mathrm{mV}$. None occurred. The battery was at $30 \% \mathrm{SoC}$.

b) Nine days later, the battery was charged, discharged, and recharged to $30 \% \mathrm{SoC}$, with infamily individual cell module voltage measurements throughout.

c) The pre-test capacity cycling test was performed 13 days prior to the vibration testing, and results very consistent with the post test capacity.

d) If 2 internal shorts developed during vibration, 
they must have been of high enough impedance to escape detection during the capacity cycling, and then, transitioned to low impedance shorts to cause the thermal damage on the corners of cell $\# 4$ and \#5. These two shorts must have occurred at nearly the same time or else the first short would have consumed the cell module energy.

e) No clear path of heat affected zones exists between the two corners that indicates that one caused the other.

\section{Root Cause Validation}

To validate the folded seal impingement theory, fully charged cells from the same lot as the flight lot were drop tested on their bottom corners from up to $1.5 \mathrm{~m}$ high. When the seal is folded as in the cell\#5 configuration, the pouch is not breached, even though the corners of cells are very deformed. Surprisingly, no anomalous OCV decay occurred. When the cell seal is not folded, drops from over $60 \mathrm{~cm}$ cause corner pouch tears. Two-cell stacks representing cell $\# 4$ adjacent to cell $\# 5$ are planned to be dropped to further investigate this theorized root cause.

To validate the cold flow of the insulating plastic layers of the cell pouch, cold flow force measurements are planned with the laminate pouch materials.

To validate the misalignment of the electrode theory, defects in the foam of engineering cell modules were introduced at the corners. These defective modules were assembled into engineering batteries and vibrated very similarly to the flight batteries. So far, no anomalous decay in cell module OCVs have occurred.

No plan has yet been developed to replicate the native material contamination theory.

\section{Corrective Actions}

The current acceptance screening for the internal shorts $[1,2]$ is only adequate at the cell level. Current procedures are inadequate at the cell module level because no thermal cycling or vibration is followed with capacity cycling tests with appropriate hold times.

Screening for internal shorts at the completed flight battery assembly level is a success oriented approach which high risk when a failure is detected.

The proposed corrective actions are to perform thermal cycle and vibration at the cell module level. This should be followed with capacity cycling tests that include hold times designed to detect high self-discharge and soft shorts. Short shorts are detected by fully discharging a cell module at $3.0 \mathrm{~V}$ to a $125 \mathrm{~mA}$ taper current and monitoring cell open circuit voltage on day 14 and 21 of the hold time. Any cell module whose voltage is declining between day 14 and 21 is rejected. At this low state of charge, a great majority of soft shorts will produce a measurable OCV decay. Once the cell modules are cleared for assembly into batteries, battery thermal cycling and vibration acceptance testing can be done at levels for detecting standard workmanship defects rather than the higher levels that had been used to screen for internal shorts.

\section{REFERENCES:}

[1] Bhola, R., "Cell Acceptance and Lot Certification Plan, EV307715, July 2005.

[2] Bhola, R., "Cell Acceptance and Lot Certification Procedure, EV307716, July 2005.

\section{Acknowledgements}

The author thanks the contributions of John Weintritt and Dan Hall of Jacobs Engineering Group and Rakesh Bhola and Jim Jacobs of Electrovaya to this failure investigation.

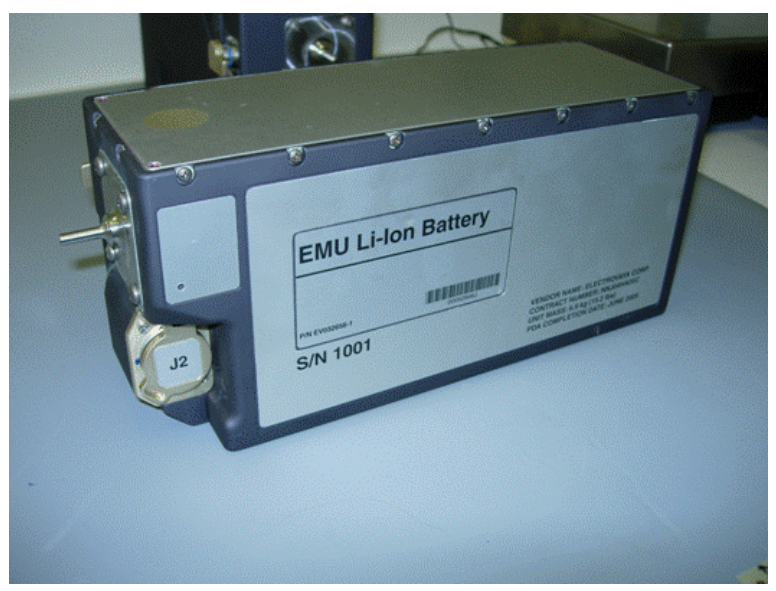

Figure 1. Lithium Ion Battery for the Spacesuit.

LIB BATTERY

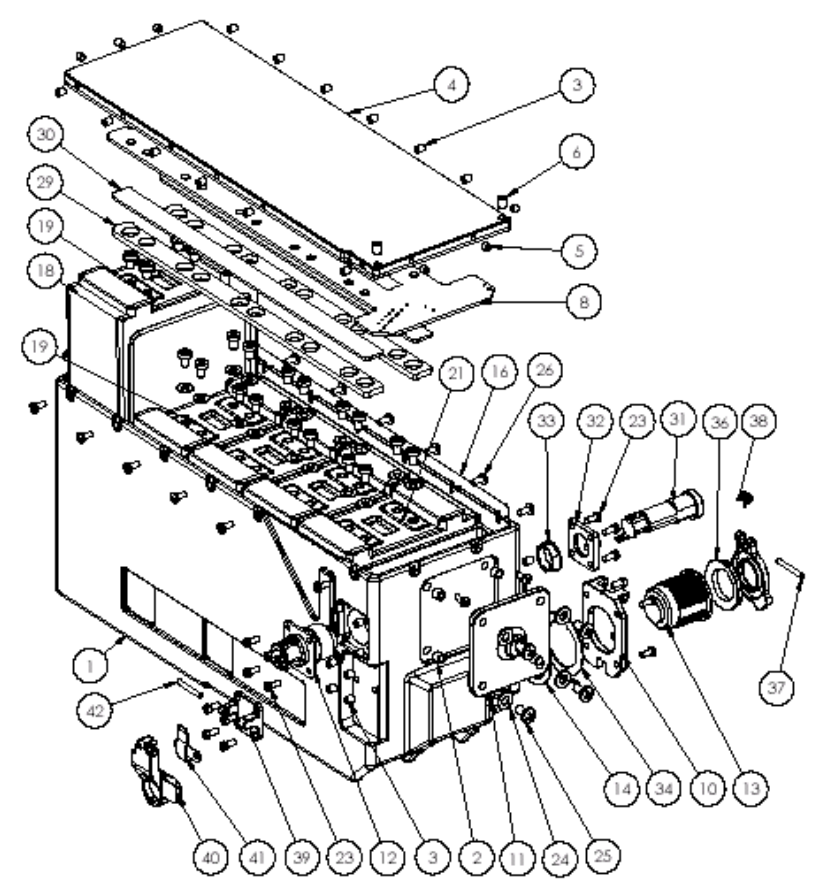

Figure 2. Expanded drawing of the Spacesuit Battery

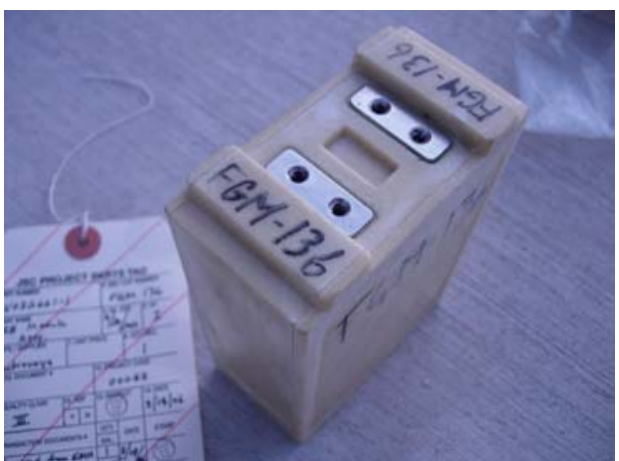

Figure 3. One of 5 cell modules in a Spacesuit Battery

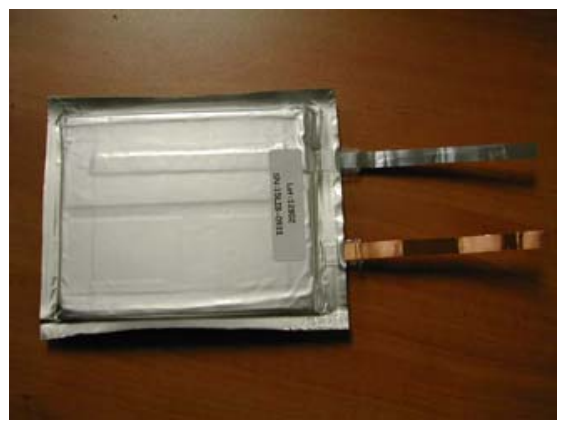


Figure 4 . One of the 5 cells that make up a cell module

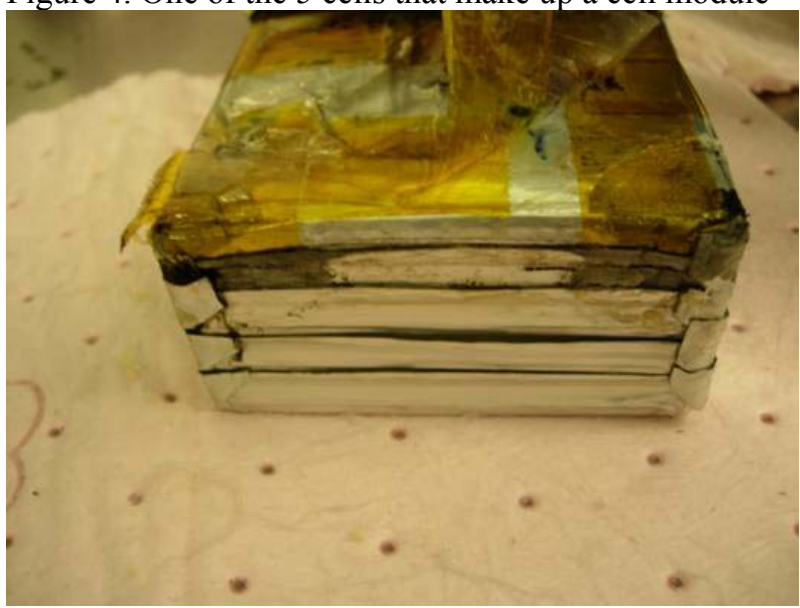

Figure 5. The five-cell stack of the internally shorted cell module after outer pouch was removed. Note the heat affected bottom corners of cells $4 \& 5$.

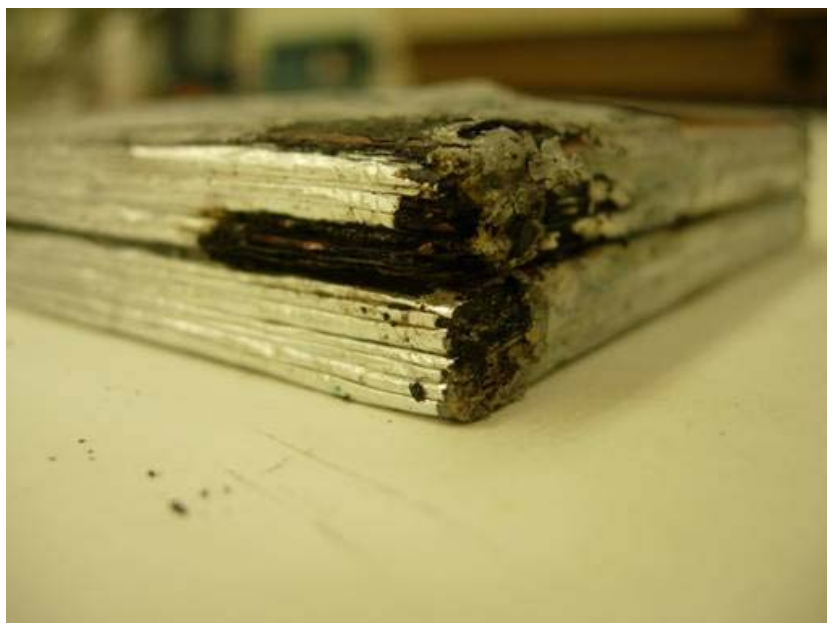

Figure 6. Damaged bottom corner of Cell \#5 on top of Cell \#4 with cell pouches removed.

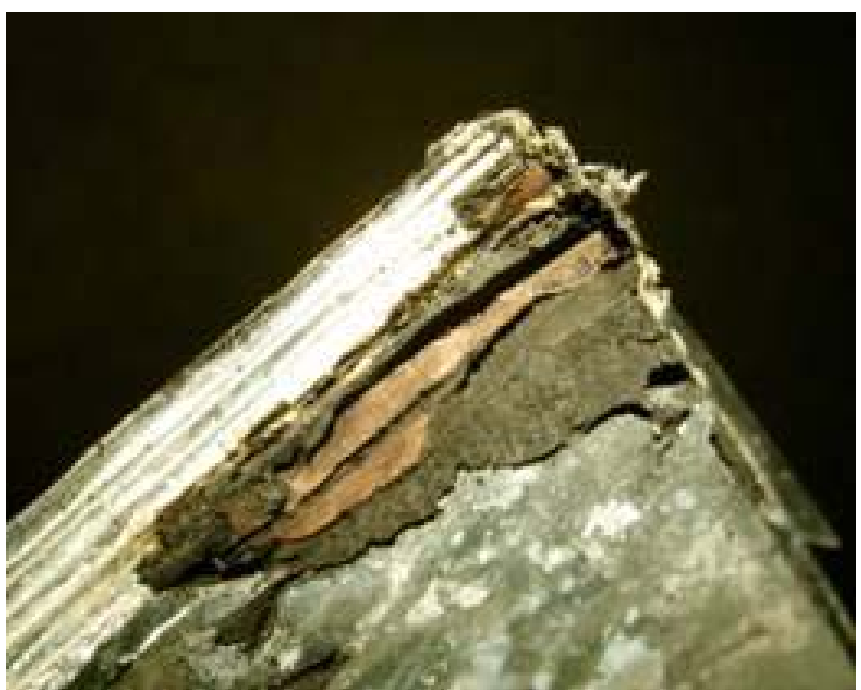

Figure 7. Torn copper substrate folds exposed on the bottom corner of cell $\# 5$.

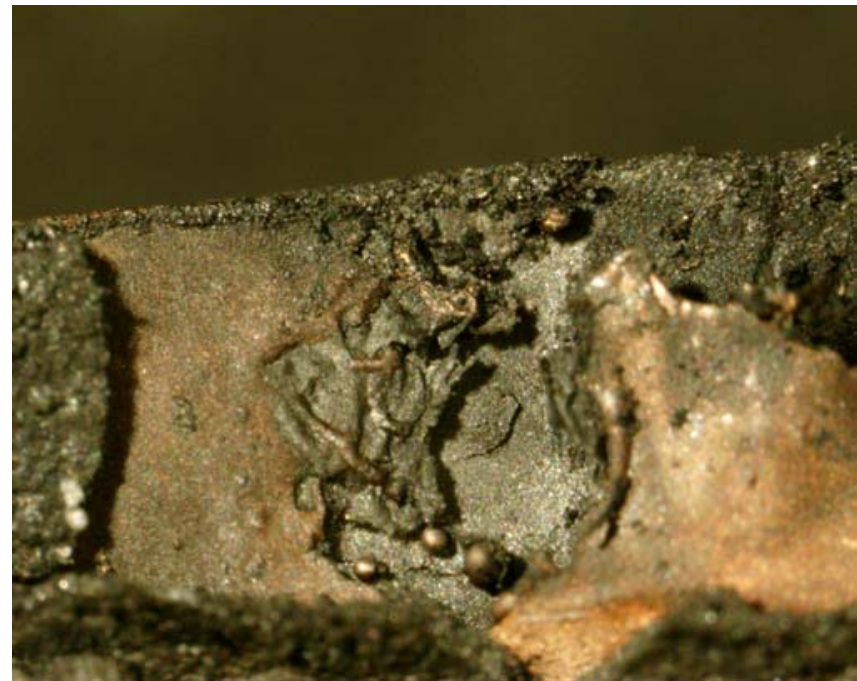

Figure 8. Ground zero arc damage on the copper foil substrate on a torn side fold near the bottom corner of cell \#5.

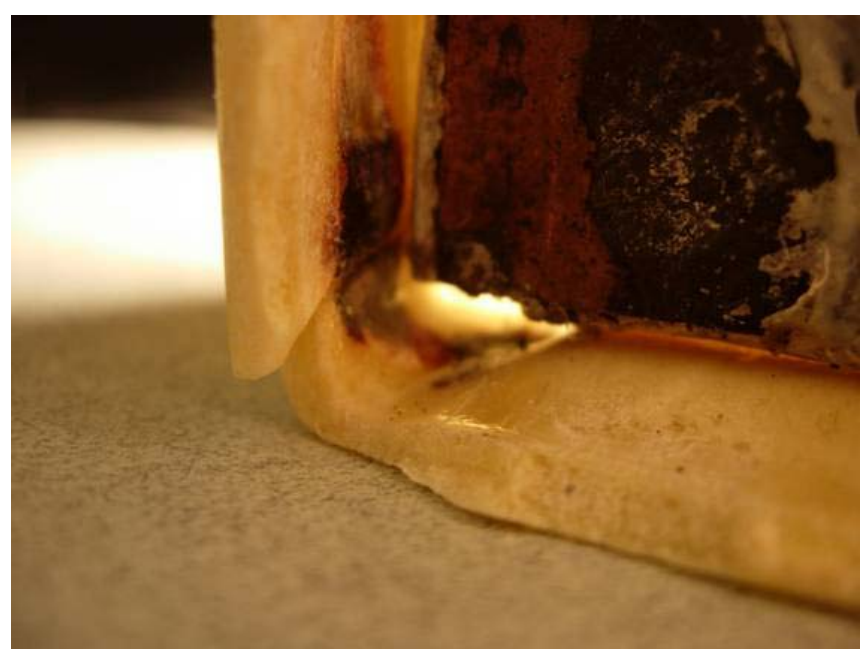

Figure 9. Cell module urethane foam with cell $\# 4$ in its position. This shows the non-uniform thickness of the foam layers near the bottom corner.

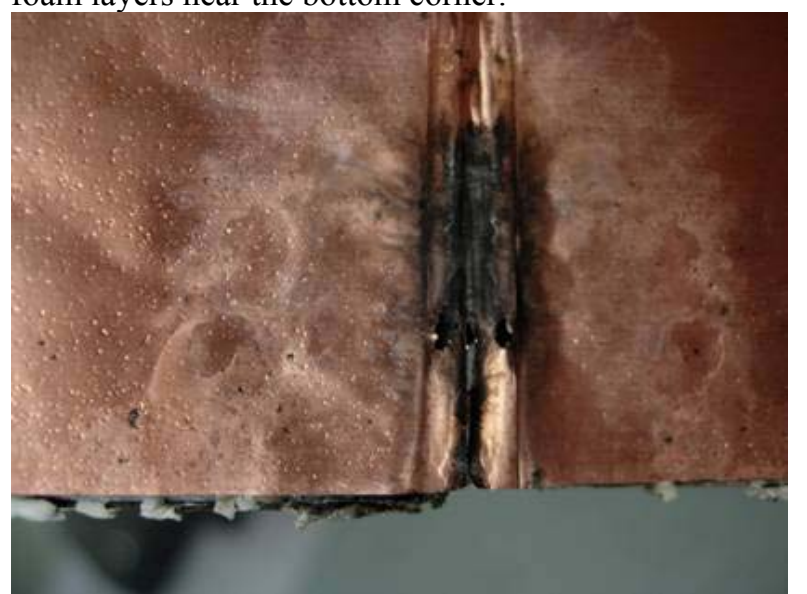

Figure 10. Unfolded cell \#5 stack showing the torn copper folds and ground zero of the internal short. 


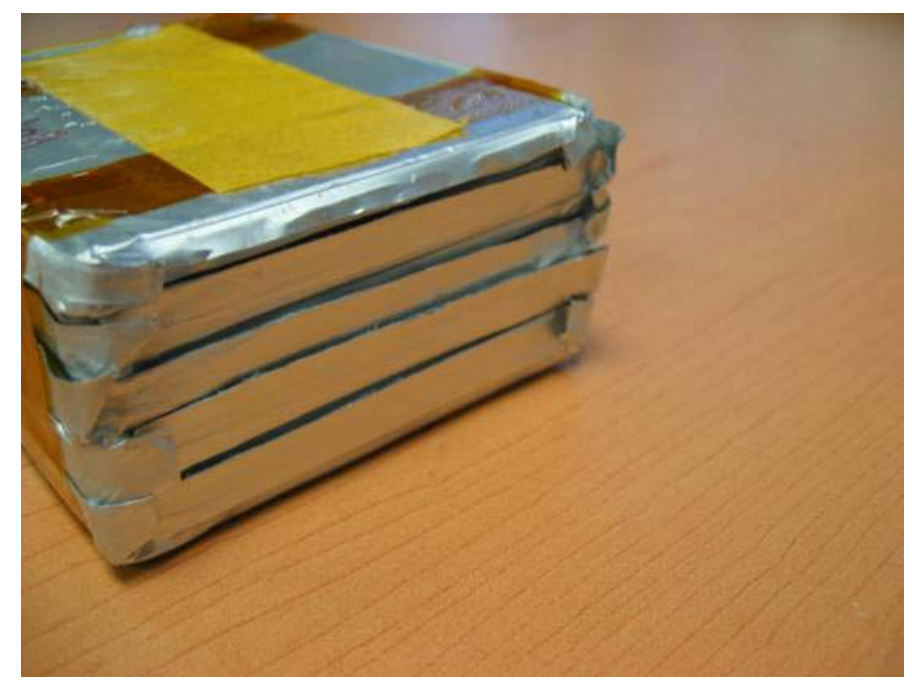

Figure 11. Bottom of a nominal 5-cell stack of a cell module, showing how the edge and corner seals are

folded. Note how the edge seal of the top cell (\#5) is

folded differently than the seal of the other cells. 